\title{
Harina De Ají De Ratón (Capsicum Mínimum) Como Anticoccidial Natural En Pollos De Engorde, Manabí-Ecuador
}

\author{
Francisco Javier Oñate, \\ Luis Fiallos, \\ Nelson Duchi, \\ Alex Villafuerte, \\ Isabel Peñafiel, \\ Ángel Flores,
}

Escuela Superior Politécnica de Chimborazo

Klever Morales,

Escuela Superior Politécnica Agropecuaria de Manabí

Doi: 10.19044/esj.2018.v14n12p15 URL:http://dx.doi.org/10.19044/esj.2018.v14n12p15

\begin{abstract}
The use of natural plants for the control and treatment of diseases is an alternative to drugs and antibiotics. In the present test the hot pepper (Capsicum Minimum) was used as an anticoccidial in broiler chickens, it was taken as a reference to salinomycin (control) including $350 \mathrm{~g} /$ ton, faced with three increasing levels of hot pepper flour $(350,500$ and $1000 \mathrm{~g} /$ ton). For the effect, 160 newborn chicks of the Cobb 500 line were used, distributed in four treatments and four repetitions under a completely randomized design, for the comparison of means the discrete Tukey test $(0,05)$ was used, the data were analysed in the free version of InfoStat 2014 program. The variables analysed were weight, feed consumption, feed conversion, mortality and counting of Eimeria oocysts in faeces. Regarding the weight, the treatments presented statistical equality ( $p>0.05)$, numerically T3 registered the highest $(2405.13 \pm$ 43.33); and food conversion T1 and T2 (1.69), T3 and T4 (1.80) did not present differences between their means. Regarding mortality, T3 did not present deaths, not so, T4 (2.5\%); T2 (5\%) and T1 (10\%); for oocyte count of E. Tenella and Acervulina_T3 and T4 do not register eggs, T1 (3000 and 4500) and T2 (2500 and 1500, respectively). The hot pepper flour has anticoccidial activity in broiler chickens, its inclusion did not alter the productive parameters of the flock, which is recommended to be used during the fattening stage of the chickens or fowls.
\end{abstract}


Keywords: Capsicum, hot pepper, coccidia, anticoccidial

\section{Resumen}

El uso de plantas naturales para el control y tratamiento de enfermedades constituyen una alternativa a drogas y antibióticos. En el presente ensayo se utilizó harina de ají de ratón (Capsicum minimum) como anticoccidial en aves de ceba, se tomó como referencia a la salinomicina (testigo) con inclusión de $350 \mathrm{~g} / \mathrm{ton}$, enfrentada a tres niveles crecientes de harina de ají (350, 500 y $1000 \mathrm{~g} /$ ton). Parta el efecto se utilizaron 160 pollitos recién nacidos de la línea Cobb 500 distribuidos en cuatro tratamientos y cuatro repeticiones bajo un diseño completamente aleatorizado, para la comparación de medias se utilizó la prueba honesta de Tukey $(0,05)$, los datos fueron analizados en el programa en versión libre InfoStat 2014. Las variables analizadas fueron peso, consumo de alimento, conversión alimenticia, mortalidad y conteo de oocitos de Eimeria en heces. En cuanto al peso los tratamientos presentaron igualdad estadística $(\mathrm{p}>0,05)$, numéricamente T3 registró el más alto $(2405,13 \pm 43,33)$; y conversión alimenticia T1 y T2 $(1,69)$, T3 y T4 $(1,80)$ tampoco presentó diferencias entre sus medias. Respecto a la mortalidad T3 no presentó muertos, no así, T4 (2,5\%); T2 (5\%) y T1 (10\%); para el conteo de oocitos de E. tenella y acervulina T3 Y T4 no registran huevos, T1 (3000 y 4500) y T2 (2500 y 1500, respectivamente). La harina de ají presenta actividad anticcocidial en aves de engorde, su inclusión no alteró los parámetros productivos de la parvada, por lo cual se recomienda utilizarla durante la etapa de engorde de las aves.

Palabras claves: Capsicum, ají de ratón, coccidia, anticoccidial

\section{Introducción}

La coccidiosis en una enfermedad producida por protozoarios microscópicos del genero Eimeria que suelen multiplicarse en las células epiteliales del intestino, su presencia produce efectos económicamente negativos en las industrias ganaderas vacunas, ovinas, porcinas y en aves de corral (Alcaíno et al., 2002). Los pollos de engorde contagiados con coccidia tienen serios problemas con la absorción de vitaminas y nutrientes; además, la pigmentación de su carcasa se puede apreciar muy disminuida ya que se presentan lesiones muy serias a nivel de intestinos lo que retarda el desarrollo de las aves llegando incluso a presentar altas mortalidades (Deepa et al 2007).

Durante décadas se han ideado distintos métodos para controlar la coccidiosis aviar; los primeros medicamentos utilizados fueron las sulfamidas, posteriormente el mercado probó moléculas como los nitrofuranos, de uso prohibido en la actualidad. Largos estudios produjeron y desarrollon antibióticos obtenidos por síntesis fermentativa (salinomicina, monensina, 
narasina, lasalocid) y compuestos de síntesis química (diclazuril, nicarbazina, halofuginona) (Yuño y Gogorza, 2008). La principal característica de los ionoforos radica en que no han demostrado interferencia alguna con la inmunidad, por lo cual se utilizan combinaciones de ionoforos y químicos. Los anticoccidiales sin excepción son susceptibles de inducir resistencia por parte de los protozoarios, se han observado resistencias en campo (E. Acervulina, $E$. Maxima, E. Tenella) ante drogas ionoforas, se recomienda realizar rotación para evitar las resistencias (Anderson at al., 2005).

Existe interés por el desarrollo de alternativas novedosas que se ocupen del control de la coccidia en aves, hay que tomar en cuenta que se hallan en boga políticas que restringen el uso de antibióticos así como la quimioterapia profiláctica en animales cuyo destino sea el consumo humano (Anderson et al., 2005; Yuño y Gogorza, 2008).

Por miles de años se han utilizado plantas y extractos vegetales para el control de patologías, se han logrado descubrir propiedades únicas en especies vegetales que contrarrestan la actividad microbiana, bacterial y fúngica; el ají (Capsicum sp.) es una hortaliza originaria de América adaptada a una amplia gama de pisos climáticos, poseedora de propiedades antioxidantes y antibacterianas gracias a la capsaicina y dihidrocapsaicina (capsaicinoides) presentes en su interior, estos compuestos son responsables de la pungencia y la activación de neurotransmisores que estimulan los receptores de lengua y boca. Se han estudiado varios microorganismos que se han visto inhibidos bajo presencia y contacto con el ají, la importancia de su uso radica en que las bacterias no generan resistencia alguna (Castro y Fontes, 2005; Bae et al., 2012; Cerón et al., 2014).

\section{Materiales y métodos}

\section{Localización y duración del experimento}

El experimento, se llevó a cabo en el sitio El Limón, Parroquia Pedro Ángel Giler, provincia de Manabí, Ecuador; a una altitud de 15 m.s.n.m., temperatura media anual de $26{ }^{0} \mathrm{C}$ y una humedad relativa de $73 \%$. El tiempo de ejecución fue de seis semanas.

\section{Material experimental}

Se emplearon un total de 160 pollitos recién nacidos de la línea Cobb 500 sin sexar (machos y hembras por igual).

\section{Instalaciones y equipos}

Se utilizó un galpón elevado de $30 \mathrm{~m}^{2}$, subdividido en 16 compartimientos de un metro cuadrado $\left(1 \mathrm{~m}^{2}\right)$ cada uno, las divisiones se construyeron con madera y malla. Se utilizó tamo de arroz para la cama, cada unidad estuvo provista de un comedero y bebedero. 


\section{Análisis estadístico}

Se utilizó un diseño completamente aleatorizado (DCA), con cuatro tratamientos y cuatro repeticiones cada uno, cada repetición albergaba diez pollitos, distribuidos todos al azar. Se procedió al análisis de varianza (ANAVA), utilizando el programa estadístico en versión libre Infostat (2015), el efecto significativo de las medias de las variables estudiadas $(\mathrm{p}<0.05)$ fue comparado con la prueba de Tukey.

\section{Tratamientos experimentales}

En el experimento se propuso comparar la capacidad anticoccidial de la harina de ají (Capsicum mínimum) en tres dosis de inclusión frente a un testigo comercial (salinomicina).

\begin{tabular}{ccc} 
Cuadro 1. Tratamientos experimentales \\
\hline Tratamiento & Código & $\begin{array}{l}\text { Inclusión } \\
\text { (g/ton) }\end{array}$ \\
\hline Salinomicina & T1 & 350 \\
Harina de ají & T2 & 350 \\
Harina de ají & T3 & 500 \\
Harina de ají & T4 & 1000 \\
\hline
\end{tabular}

\section{Variables evaluadas}

Las variables evaluadas fueron: peso, ganancia de peso, conversión alimenticia, mortalidad y conteo de oocitos de Eimeria.

\section{Procedimiento}

\section{Preparación de la muestra}

Se recolectaron muestras de ají de ratón (Capsicum Minimun) en la zonas aledañas a Calceta, cantón Bolívar, Provincia de Manabí. Las muestras fueron lavadas con abundante agua con el fin de eliminar residuos de arcilla $u$ otros contaminantes que podían adherirse al fruto. El material vegetal fue expuesto al sol para quitar el exceso de agua y finalmente fue llevado a una estufa a $60^{\circ} \mathrm{C}$ para su deshidratación, para luego ser triturado en un molino de alimentos.

\section{Adecuación del galpón}

El galpón fue lavado con abundante agua y detergente, posteriormente cuando había secado se desinfectó con una solución yoda en una relación 1:100 de agua. Se colocó la cama de tamo de arroz e igualmente de procedió con la desinfección.

\section{Recepción de los pollitos}

El día del ingreso de los pollitos se verificó su homogeneidad y buen 
estado físico, se registró un peso promedio de 42,25 ( $\pm 0,95 \mathrm{~g})$. El galpón mantuvo una temperatura promedio de $35^{\circ} \mathrm{C}$ para la recepción de la parvada, se ofreció alimento inicial y agua con vitaminas, ambos a voluntad.

\section{Alimentación de los pollos}

Se utilizó alimento propio, formulado bajo las recomendaciones nutricionales del manual Cobb 2015. Los animales fueron alimentados durante parte de la tarde (16h00) y durante la noche hasta la mañana siguiente para evitar el estrés por calor; su alimentación fue a voluntad, partiendo siempre de un peso conocido en la tarde y por la mañana se realizaba el pesaje del sobrante.

\section{Calendario de vacunas}

Se elaboró un calendario vacunal de acurdo a la zona (Gumboro y New Castle) a los cuatro días de edad con refuerzo a los once. Al día dieciocho se realizó un desafío con coccidia para medir la eficiencia de los anticoccidiales en estudio.

\section{Conteo de oocitos (Eimeria)}

Se utilizó el método de flotación, con solución azucarada saturada de SHEATHER modificada en el laboratorio de Química de la Carrera de Medicina Veterinaria de la Escuela Superior Politécnica Agropecuaria de Manabí, para lo cual se emplearon $1250 \mathrm{~g}$ de azúcar, $2 \mathrm{~g}$ de fenol y $1000 \mathrm{cc}$ de agua destilada, Se trabajó con el método propuesto por Espinoza (2003); se pesaron entre 2 y 5 gramos de heces y se lo colocaron en un mortero, luego se agregaron $50 \mathrm{ml}$ de solución fenolada y seguidamente se homogenizó la mezcla , a continuación se coló la solución en un vaso de precipitación, dejándola en reposo por 30 minutos, luego se procedió a colocar una lámina porta objeto sobre la solución en reposo, con el fin adherir parásitos a la placa. Finalmente se llevaron las muestras al microscopio y se observaron a través de una lente de $10 \mathrm{X}$.

\section{Resultados y discusión \\ Peso de los pollos}

El cuadro 2 reporta el análisis de varianza del peso de pollos de engorde, en la primera semana de edad se presentaron diferencias altamente significativas ( $\mathrm{p}<0.01$ ), la inclusión de harina de ají en dosis de 350 y $500 \mathrm{~g} /$ ton (T2 y T3) comparten grupo con el tratamiento testigo (T1) correspondiente al anticoccidial comercial salinomicina (350g/ton), resultados que concuerdan con Márquez et al (2003), quienes aseguran que la capsaicina presente en el ají actúa coma antibacteriano y promotor de crecimiento natural lo cual contribuye a mejorar los parámetros productivos de las aves. 
Cuadro 2. Peso semanal (g) de pollos alimentados con harina de ají de ratón como anticoccidial natural.

\begin{tabular}{|c|c|c|c|c|c|c|}
\hline \multirow{3}{*}{ Tratamientos } & \multicolumn{5}{|c|}{ Semana } & \multirow[b]{2}{*}{6} \\
\hline & 1 & 2 & 3 & 4 & 5 & \\
\hline & $* *$ & n.s & n.s & n.s & n.s & n.s \\
\hline $\mathrm{T} 1$ & $166,00 \mathrm{ab}$ & 450,84 & 886,25 & 1442,92 & 1961,86 & 2338,38 \\
\hline $\mathrm{T} 2$ & $173,63 \quad a$ & 458,88 & 885,26 & 1413,46 & 1933,68 & 2336,67 \\
\hline $\mathrm{T} 3$ & $176,63 \quad a$ & 450,13 & 853,00 & 1397,63 & 1901,15 & 2405,13 \\
\hline $\mathrm{T} 4$ & $160,50 \quad b$ & 434,1 & 857,75 & 1381,63 & 1925,26 & 2385,81 \\
\hline $\mathrm{EE}$ & 3,55 & 7,8 & 14,45 & 25,14 & 38,76 & 43,33 \\
\hline p-valor & 0,0064 & 0,1555 & 0,2221 & 0,3868 & 0,7572 & 0,5921 \\
\hline
\end{tabular}

Promedio con letras distintas en la columna, difieren significativamente según la prueba de tukey al $5 \%$ de probabilidad. n.s. No significativo.

* Diferencia significativa al 5\%

** Diferencia altamente significativa al $1 \%$.

E.E. Error estándar

Transcurrida la tercera semana no se encontraron diferencias estadísticas entre los tratamientos, se registraron pesos de $886,25 \mathrm{~g} \pm 14,45$ (T1); $885,26 \mathrm{~g} \pm 14,45$ (T2); $857,75 \mathrm{~g} \pm 14,45$ (T4) y $853,00 \mathrm{~g} \pm 14,45$ (T3), superiores comparados a los reportados por Rosero et al (2012), quienes obtuvieron $842,50 \mathrm{~g}$ en pollos Cobb 500 alimentados con balanceado comercial. Finalizada la etapa de engorde, en la sexta semana no se halló diferencia alguna entre las medias de los tratamientos estudiados, resultando numéricamente superior $\mathrm{T} 3(2405,13 \mathrm{~g} \pm 43,33)$, los cual indica que la capsaicina presente en la harina de ají mejora la absorción de los nutrientes presentes en la dieta (Lozada, 2014).

\section{Ganancia de peso}

El cuadro 3 reporta la ganancia de semanal, evaluada la primera semana se observa diferencia altamente significativa $(\mathrm{p}<0.001)$, resultando ser mejores T3 $(135,63 \mathrm{~g} \pm 3,55), \quad \mathrm{T} 2 \quad(129,63 \mathrm{~g} \pm 3,55)$ y $\mathrm{T} 1 \quad(124,00 \mathrm{~g} \pm 3,55)$, tratamientos que comparten grupo y superan a T4 $(117,50 \mathrm{~g} \pm 3,55)$, resultados similares a los reportados por Márquez et al (2003), quienes aseguran que los extractos naturales mantienen actividad antimicrobiana la cual se ve reflejada en la ganancia de peso de las aves.

Iniciada la segunda semana y hasta el final del ensayo se encontró igualdad estadística entre los tratamientos ( $\mathrm{p}>0.05)$, resultados y tendencias similares a los expuestos por Zhang et al (2005) y Pickler et al (2011), quienes aseguran haber observado un desempeño zootécnico aumentado en pollos de engorde tras el uso de capsaicina como promotor de crecimiento. 
Cuadro 3. Ganancia de peso semanal (g) de pollos alimentados con harina de ají de ratón como anticoccidial natural.

\begin{tabular}{|c|c|c|c|c|c|c|}
\hline \multirow[t]{3}{*}{ Tratamientos } & \multicolumn{6}{|c|}{ Semana } \\
\hline & 1 & 2 & 3 & 4 & 5 & 6 \\
\hline & $* *$ & n.s & n.s & n.s & n.s & n.s \\
\hline $\mathrm{T} 1$ & $124,00 \mathrm{ab}$ & 284,84 & 432,67 & 549,86 & 542,35 & 545,61 \\
\hline $\mathrm{T} 2$ & $129,63 \mathrm{ab}$ & 285,25 & 426,41 & 530,79 & 557,94 & 531,18 \\
\hline $\mathrm{T} 3$ & $135,63 \quad \mathrm{a}$ & 273,50 & 402,63 & 550,28 & 599,94 & 553,55 \\
\hline $\mathrm{T} 4$ & $117,50 \quad b$ & 273,63 & 424,75 & 539,15 & 618,61 & 633,57 \\
\hline $\mathrm{EE}$ & 3,55 & 8,09 & 16,10 & 28,85 & 40,57 & 44,81 \\
\hline$p$-valor & 0,0033 & 0,5700 & 0,5867 & 0,9627 & 0,5111 & 0,3636 \\
\hline
\end{tabular}

Promedio con letras distintas en la columna, difieren significativamente según la prueba de tukey al 5\% de probabilidad.

n.s. No significativo.

* Diferencia significativa al 5\%

** Diferencia altamente significativa al $1 \%$.

E.E. Error estándar

\section{Conversión alimenticia}

En el cuadro 4, se reporta los valores de conversión alimenticia acumulada, no se registraron diferencias significativas $(\mathrm{p}>0.05)$ para este parámetro durante el ensayo. En la primera semana T3 presenta una conversión alimenticia de $0,77( \pm 0,03)$; resultados que difieren de los presentados por Oñate et al (2016), quienes registraron una eficiencia de $0,72 \pm 0,002$ al utilizar metionina de origen herbal en reemplazo a la sintética en engorde de pollos parrilleros.

Cuadro 4. Conversión alimenticia evaluada con la inclusión de tres niveles de harina de ají en dos densidades poblacionales de pollos.

\begin{tabular}{ccccccc}
\hline & \multicolumn{7}{c}{ Semana } \\
\cline { 2 - 7 } Tratamientos & 1 & 2 & 3 & 4 & 5 & 6 \\
\cline { 2 - 7 } & n.s & n.s & n.s & n.s & n.s & n.s \\
\hline T1 & 0,88 & 1,07 & 1,35 & 1,46 & 1,63 & 1,69 \\
T2 & 0,83 & 1,03 & 1,29 & 1,46 & 1,56 & 1,69 \\
T3 & 0,77 & 1,03 & 1,35 & 1,44 & 1,76 & 1,80 \\
T4 & 0,79 & 1,1 & 1,31 & 1,42 & 1,63 & 1,80 \\
EE & 0,03 & 0,01 & 0,05 & 0,04 & 0,05 & 0,05 \\
p-valor & 0,1234 & 0,0925 & 0,1918 & 0,0481 & 0,0808 & 0,3462 \\
\hline
\end{tabular}

Promedio con letras distintas en la columna, difieren significativamente según la prueba de tukey al 5\% de probabilidad.

n.s. No significativo.

* Diferencia significativa al 5\%

** Diferencia altamente significativa al $1 \%$.

E.E. Error estándar 
En la cuarta semana T4 registra $1,42( \pm 0,04)$; y en la sexta $\mathrm{T} 1$ y T2 presentan una conversión alimenticia similar $1,69( \pm 0,05)$ diferentes numéricamente comparados con T3 y T4 $(1,80 \pm 0,05)$; resultados similares a los reportados por Gómez et al (2013) quienes aseguran que los capsaicinoides presentes en el ají estimulan el sistema inmunológico de las aves.

\section{Mortalidad}

El gráfico 1 reporta la mortalidad de las aves durante la fase experimental de campo expresada en porcentajes, la inclusión de $500 \mathrm{~g}$ de ají por tonelada de alimento (T3) no reportó muerte alguna, seguido por T4 ( $1000 \mathrm{~g} /$ ton) y T2 $(350 \mathrm{~g} / \mathrm{ton})$ con 2,5 y $5 \%$ respectivamente, superando todos al testigo (T1) que presentó una mortalidad del 10\%, estos resultados son similares a los reportados por Gómez et al (2013) y da Costa et al (2009), investigadore que aseguran que la inclusión de plantas naturales como el ají (Capsicum annum) en las dietas de las aves mejora el metabolismo de los nutrientes y su estatus inmunológico.

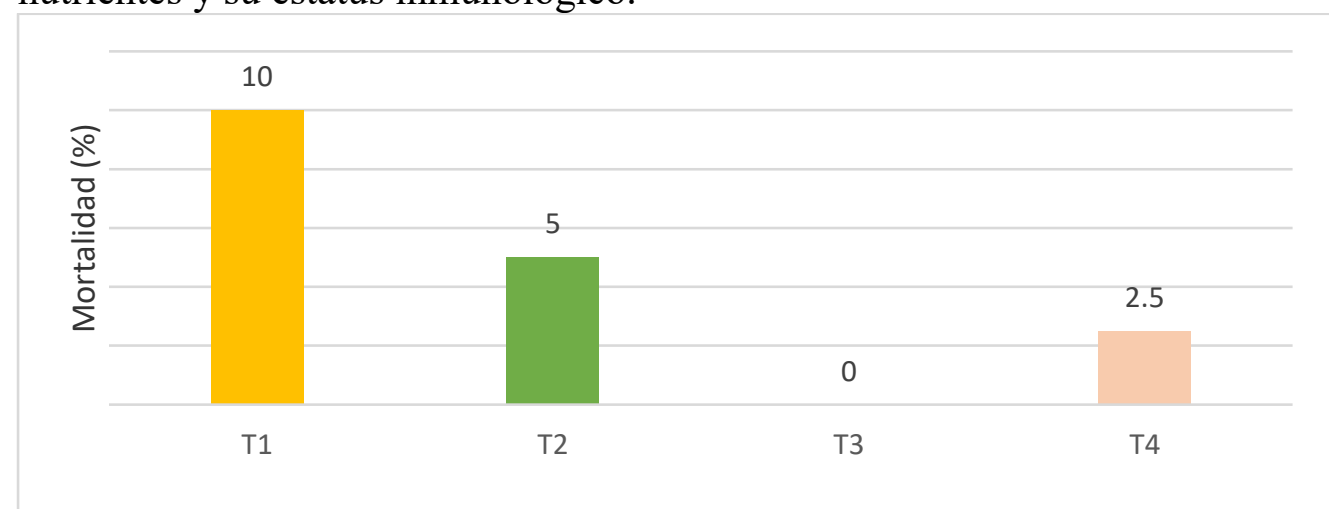

Gráfico 1. Mortalidad (\%) de las aves alimentadas con harina de ají

\section{Conteo de oocitos de Eimeria}

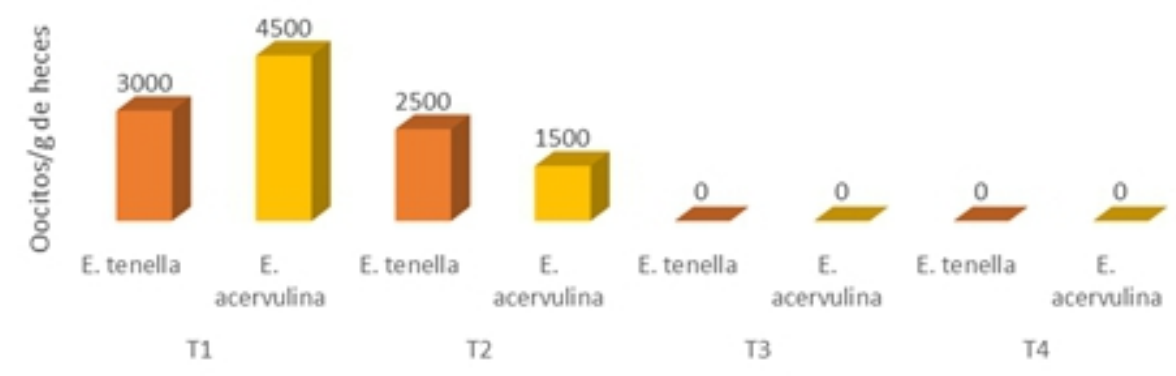

Gráfico 2. Conteo de oocitos de Eimeria Tenella y Acervulina por gramo de heces 
La grafica 2 ilustra los resultados obtenidos en la prueba coproparisitaria dentro de los 35 días de edad, se trabajó con la técnica descrita y utilizada por Espinoza (2003) quien utilizó la técnica de sedimentación cuyos resultados se expresan en oocitos por gramo de heces (OPGH). Se buscaron las Eimerias más recurrentes (E. Tenella y Acervulina) T3 y T4 no reportaron presencia de oocitos, mientras T1 $(3000 ; 4500)$ y T2 $(2500 ; 1500)$ oocitos de E. Tenella y acervulina, respectivamente; valores que se encuentran dentro de los límites permisibles según Alcaíno et al (2002) y Valladares (2010), estudiosos que indican que las Eimerias producen enfermedad cuando sobrepasan los límites permisibles E. Acervulina (100000 OPGH), E. Tenella (70000 OPGH) y E, Maxima (10000 OPGH).

\section{Conclusiòn}

La incidencia de coccidia en las aves alimentadas con inclusiones de harina de ají en la dieta se ve disminuida, lo cual es un claro indicativo que posee acción anticoccidial y coccidicida.

\section{References:}

1. Alcaíno, H., Gonzales, J., Fredes, F. y Gorman, T. 2002. Coccidias aviares de gallineros industriales en Chile. Parasitologìa latinoamericana. 57(12):34-39.

2. Anderson, J., Bftu, T., Brown, C., Donald, R., Gurnett, A., Leavitt, P., Mathew, J., Bakela, N., Schematz, D., Tamas, T., Thompson, D., Zhong, T. and Liberator, P. 2005. Anticoccidial drugs discovery: approaches toward the identification of novel chemotherapeutic agents. Proceedings of The International Coccidiosis Conference, Foz do Iguazù, Brasil.

3. Bae, H., Jayaprakasha, G., Jifon, J. and Patil, B. 2012. Variations of antioxidant activity and the levels of bioactive compunds in lipophilic ans hydrophilic extracts from hot pipper (Capsicum spp.) cultivars. Food Chemistry. 134:1912-1918.

4. Castro, M. and Fontes, W. 2005. Plants defense and antimicrobial peptides. Protein Pept. Lett. 12(1):8-13

5. Cerón, T., Munguía, R., García, S. y Santiesteban, A. 2014. Actividad antimicrobiana de extractos de diferentes especies de chile (capsicum). Revista Iberoamericana de Ciencias. 1(2):213-221.

6. Cobb Nutrition Supplement. 2015. Aviagen. Newbridge. Scotdland.

7. Costa da, L., Moura de, N., Marangoni, C., Mendes, C. y Teixeira. 2009. Actividade antioxidante de pimentas do gênero Capsicum. Ciênc.Tecnol. Aliment. 30(11):10-19.

8. Deepa, N., Charanjit, K., Binoy, G., Balraj, S. and Kapoor, H. 2007. Antioxidant constituents in some sweet pepper (Capsicum annuum L.) 
genotypes during maturity. Food Science and Technology. 40(1):121129.

9. Espinoza, A. 2003. Efecto de la Ivermectina (Ivercide) en el control de parásitos internos y externos en pollos de engorde en Zamorano. Carrera de Ciencia y Producción Agropecuaria.p 56.

10. Gómez J., Vargas, L. y Sanabria, F. 2013. Los capsaicinoides presentes en el cultivo de ají como estimulante del sistema inmunológico en aves. Colección Selección de Investigaciones. $N^{0} 6$.

11. Lozada, J. 2014. Evaluación del ají (capsicum annuum) como aditivo natural para la prevención de coccidiosis en pollos parrilleros. Facultad Ciencias Agropecuarias. Tesis. Medicina Veterinaria y Zootecnia. Universidad Técnica de Ambato.

12. Márquez, D., Galeano, E. y Martínez, A. 2003. Productos naturales con actividad antimicrobiana. Revista de la Facultad de Química Farmacéutica. Universidad de Antioquia-Colombia. 10(2):61-71.

13. Oñate, F., Larrea, C. y Paredes, M. 2016. Efecto de la metionina herbal sobre el rendimiento productivo en pollos parrilleros. Espamciencia. 7(19:37-41-

14. Pickler, L., Santin, E. y Fischer, A. 2011. Alternativas aos antibióticos para equilibrar a microbiota gastrointestinal de fragos. Archives of Veterinary Science. 16(3):1-13.

15. Rosero, J., Guzmán, E. y López, F. 2012. Evaluación del comportamiento productivo de las líneas de pollos de engorde Cobb 500 y Ross 308. Biotecnología en el sector Agropecuario y Agroindustrial. 10(1):8-15.

16. Valladares, J. 2010. Elementos requeridos para un diagnóstico de Laboratorio. Art. 2: 3-8. Asesoría Avícola Independiente.

17. Yuño, M. y Gorgorza, L. 2008. Coccidiosis aviar: respuesta inmune y mecanismos de control en la industria avícola. Rev. Vet. 19(1):61-66.

18. Zhang, H., Yan, F., Keen, C. and Waldroup, P. 2005. Evaluation of microencapsulated essential oils and organic acids in diets of broilers chickens. International Journal of Poultry Science. 4(9):612-619. 